\title{
Review: lipid lowering drugs decrease coronary artery disease (CAD) events but not all cause or CAD mortality in men with no history of CAD
}

\author{
Pignone M, Phillips C, Mulrow C. Use of lipid lowering drugs for primary prevention of coronary heart disease: meta \\ analysis of randomised trials. BMJ 2000 Oct 21;321:983-6.
}

QUESTION: In patients with no history of coronary artery disease (CAD), does a lipid lowering drug (cholestyramine, gemfibrozil, pravastatin, or lovastatin) decrease the risk for CAD events, CAD mortality, or all cause mortality?

\section{Data sources}

English language studies were identified by searching Medline (1994 to June 1999) with the Medical Subject Headings (MeSH) hyperlidemia and anti cholesterolemic agents and with MeSH or keywords identifying randomised controlled trials and individual drug names, the Cochrane Clinical Trials Registry, and bibliographies of systematic reviews and clinical practice guidelines.

\section{Study selection}

Randomised controlled trials were selected if they had $\geqslant 1$ year of duration and measured CAD events, CAD mortality, and all cause mortality. Exclusion criteria were studies of secondary prevention or studies published in abstract form only.

\section{Data extraction}

Data were extracted on each drug and its dosage, study duration, population characteristics and size, initial and mean reduction in cholesterol concentrations, CAD events, CAD mortality, and all cause mortality.

\section{Main results}

4 randomised controlled trials met the inclusion criteria. 21087 patients (mean age 53 y, 95\% men) received a lipid lowering drug $(50 \%)$ or placebo $(50 \%)$. Duration was 7 years ( 1 study) or 5 years ( 3 studies). The mean initial total cholesterol concentration was $\geqslant 7.0 \mathrm{mmol} / \mathrm{l}$ in 3 of the 4 trials. Mean reductions in cholesterol concentrations were $8.5 \%$ for cholestyramine, $10 \%$ for gemfibrozil, and $18 \%$ to $20 \%$ for high dose statins. Treatment decreased CAD events but not CAD mortality or all cause mortality (table). When analysis was limited to the 2 trials that used statins, the results were similar.

\section{Conclusion}

In patients with no history of coronary artery disease (CAD), lipid lowering drugs decrease the risk for CAD events but not CAD mortality or all cause mortality.

Lipid lowering drugs (LLDs) v placebo for decrease of coronary artery disease (CAD) events and mortality and all cause mortality in patients with no history of $C A D^{*}$

\begin{tabular}{|c|c|c|c|c|}
\hline \multirow[b]{2}{*}{ Outcomes at median 5 years } & \multicolumn{2}{|c|}{ Unweighted event rates } & \multirow{2}{*}{$\begin{array}{l}\text { RRR }(95 \% \text { CI) } \\
\text { weighted }\end{array}$} & \multirow{2}{*}{$\begin{array}{l}\text { NNT (Cl) } \\
\text { weighted }\end{array}$} \\
\hline & LLDs & Placebo & & \\
\hline CAD events in all 4 studies & $4.2 \%$ & $5.8 \%$ & $28 \%(19$ to 36$)$ & 69 (49 to 120$)$ \\
\hline CAD events in 2 statin studies & $3.5 \%$ & $5.2 \%$ & $33 \%(21$ to 43$)$ & $69(49$ to 120$)$ \\
\hline CAD mortality in all 4 studies & $0.9 \%$ & $1.2 \%$ & $25 \%(2$ to 43$)$ & Not significant \\
\hline All cause mortality in all 4 studies & $2.8 \%$ & $3.1 \%$ & $8 \%(-7$ to 21$)$ & Not significant \\
\hline All cause mortality in 2 statin studies & $2.8 \%$ & $3.2 \%$ & $12 \%(-6$ to 28$)$ & Not significant \\
\hline
\end{tabular}

Source of funding: Agency for Healthcare Research and Quality.

For correspondence: Dr M Pignone, Division of General Internal Medicine, 5039 Old Clinic Building, University of North Carolina, Chapel Hill, NC 27599-7110, USA. $\mathrm{Fax}+1919966$ 2274.

\section{COMMENTARY}

The meta-analysis by Pignone et al reinforces the first rule of primary prevention for CAD: absolute reduction in mortality is proportional to overall risk for CAD. Although the authors conclude that "treatment with lipid lowering drugs ... reduces CHD [sic] events but not all cause mortality in people with no known cardiovascular disease," they also point out that the West of Scotland Coronary Prevention Study, ${ }^{1}$ whose participants had a relatively high risk for CAD $(8 \%)$, had a reduction in all cause mortality with pravastatin. Furthermore, a previous meta-analysis that did not include a trial whose participants were at very low risk also suggested that lipid lowering reduces all cause mortality. ${ }^{2}$

The meta-analysis by Pignone et al relies on high quality studies but does not give us reliable information on adverse event rates. The results of these studies may not be generalisable to other populations, particularly the elderly and women, because these demographic groups were underrepresented in the trials considered in the meta-analysis.

Lipid lowering was not shown to reduce all cause or CAD mortality in patients at low risk for CAD probably because it takes larger numbers of patients or longer trials to show small, statistically significant absolute reductions in mortality. We have no reason to believe that lipid lowering increases non-coronary mortality. Physicians should continue to consider lipid lowering for primary prevention. As pointed out by the authors, overall CAD risk should be calculated and used to select higher risk patients for treatment with lipid lowering drugs. ${ }^{3}$ For low risk patients, issues of cost effectiveness may predominate.

Edward P Havranek, MD Denver Health Medical Center Denver, Colorado, USA

1 Sheperd J, Cobbe SM, Ford I, et al. Prevention of coronary heart disease with pravastatin in men with hypercholesterolemia. N Engl J Med 1995;333:1301-7.

2 Hebert PR, Gaziano JM, Chan KS, et al. Cholesterol lowering with statin drugs, risk of stroke, and total mortality. An overview of randomized trials. JAMA 1997;278:313-21.

3 Jackson R. Guidelines on preventing cardiovascular disease in clinical practice. BMJ 2000;320:659-61. 